OPEN ACCESS

Edited by:

Mehdi Razzaghi-Abyaneh, Pasteur Institute of Iran (PII), Iran

Reviewed by: Geromy G. Moore,

United States Department of Agriculture, Agricultural Research

Service, United States

Zuzana Hruska,

Mississippi State University,

United States

${ }^{*}$ Correspondence:

Mark A. Weaver

Mark.Weaver@usda.gov orcid.org/0000-0001-9020-7264

Specialty section:

This article was submitted to

Food Microbiology,

a section of the journal

Frontiers in Microbiology

Received: 14 April 2019

Accepted: 19 July 2019

Published: 07 August 2019

Citation:

Weaver MA and Abbas HK (2019) Field Displacement of Aflatoxigenic

Aspergillus flavus Strains

Through Repeated Biological

Control Applications.

Front. Microbiol. 10:1788.

doi: 10.3389/fmicb.2019.01788

\section{Field Displacement of Aflatoxigenic Aspergillus flavus Strains Through Repeated Biological Control Applications}

\author{
Mark A. Weaver ${ }^{*}$ and Hamed K. Abbas \\ Biological Control of Pests Research Unit, United States Department of Agriculture, Agricultural Research Service, \\ Stoneville, MS, United States
}

A popular pre-harvest strategy to mitigate aflatoxin contamination of corn involves field application of non-aflatoxigenic strains of Aspergillus flavus. The basis of this biological control may involve multiple factors, but competitive displacement of aflatoxigenic strains by the biocontrol strains is a likely mechanism. Three biocontrol strains (NRRL 21882, 18543, and 30797) were applied annually, over a 4-year period, to the same 3.2-ha commercial corn field in the Mississippi Delta, where we monitored their post-release establishment, spread, and persistence. Within 2 months of the first biocontrol application, the percentage of soil-inhabiting aflatoxigenic $A$. flavus strains in some plots was reduced from 48 to $9 \%$ of the population. The frequency of aflatoxigenic $A$. flavus strains was also significantly reduced in the rest of field. After 4 years, neighboring plots that had never received a biocontrol treatment, and distanced from our treatment plots by at least 20 meters, had less than $20 \%$ aflatoxigenic isolates. This significant halo effect might be attributed to movement of soil through tillage operations, but the aflatoxigenicity shift could be detected in the untreated plots within 2 months of the initial applications, at a time when there was no tillage. The A. flavus populations that colonized the grain were also monitored and found to be less than $15 \%$ toxigenic in the fourth year for all treatments. Over all treatments and years, less than $2 \mathrm{ppb}$ of aflatoxin was detected, which could be a consequence of the field-wide shift of the inherent $A$. flavus population to predominately non-aflatoxigenic strains. This study supports the efficacy of using non-aflatoxigenic $A$. flavus strains as pre-harvest biocontrol, and shows that most of its effectiveness occurs with the first application.

Keywords: aflatoxin prevention, biopesticide, mycotoxin, biological control, atoxigenic

\section{INTRODUCTION}

Aspergillus flavus is a common soil saprophyte and an opportunistic plant pathogen. Many agriculturally important crops can be infected by A. flavus. The resulting infections rarely cause appreciable yield loss, but can be important due to the resulting contamination by A. flavus mycotoxins. Several mycotoxins can be produced by A. flavus including aflatoxin (AF) and cyclopiazonic acid (CPA). Aflatoxin AF, a Group 1 carcinogen (IARC, 1993), is produced in several forms by various fungal species, and aflatoxin $B_{1}$ is considered an especially potent liver carcinogen. Aflatoxin contamination of food and feed is especially problematic in parts of the world where the lack of testing and proper storage conditions lead to periodic 
aflatoxicosis, reduced growth in infants and children, and elevated risk of cancers (Wild et al., 2015). Europe and North America have enacted strict controls on the allowable levels of AF in foods and feeds, and the agricultural industry cooperates in monitoring AF throughout the food chain. Globally, the commodity that is most affected by AF is corn, resulting in tens to hundreds of millions of dollars in lost value annually. The level of economic loss varies greatly from year to year, but one model indicated a loss of up to US\$ 1.06 billion in 2012, when weather conditions in the upper Midwest were particularly conducive to $A$. flavus infection and subsequent AF contamination, with corn prices at or near record highs (Mitchell et al., 2016). While there is comparatively less corn grown in the Southern U.S., it is contaminated by AF more frequently and at higher concentrations (Jones et al., 1981; Diener et al., 1987; Payne, 1992; Mitchell et al., 2016).

With these strong economic incentives, corn producers endeavor to reduce AF contamination. Because A. flavus is an opportunistic pathogen, efforts to promote host plant health and minimize plant stress may be helpful, but are often insufficient (Bruns, 2003; Wiatrak et al., 2005). AF contamination linked to insect activity, especially by the European Corn Borer (Ostrinia nubilalis) and Fall Armyworm (Spodoptera frugiperda), led to the expectation that transgenic insect control would reduce AF contamination (Dowd, 2001; Abbas et al., 2013). Bt-corn, developed to prevent host damage from insect predation, has been shown to be effective in the management of other mycotoxins, e.g., fumonisin (Munkvold et al., 1999), but success with AF management by $B t$ insect control has been equivocal (Bowen et al., 2014; Weaver et al., 2017a,b). The most effective AF-reducing strategy is the pre-harvest application of $A$. flavus isolates that are naturally incapable of aflatoxin production. This strategy has been commercialized in the U.S. via A. flavus strain NRRL 21882 (AflaGuard GR, Syngenta Crop Protection, Greensboro, NC) and NRRL 18543 (AF36, Arizona Cotton Research and Protection Council), whose effectiveness has been repeatedly validated in U.S. field trials (Brown et al., 1991; Cotty, 1994; Bock et al., 2004; Dorner, 2010; Weaver et al., 2015), Europe (Mauro et al., 2018), and Africa (Atehnkeng et al., 2008; Bandyopadhyay et al., 2016).

While overall efficacy of this particular biocontrol approach for aflatoxin is well supported, questions remain regarding the multi-year dynamics of the indigenous A. flavus population in the context of biocontrol applications. We describe here an experiment involving 4 years of annual biocontrol applications, in a highly productive corn monoculture, to monitor A. flavus populations in the soil and in host kernel tissue. This experiment tested the hypotheses that applied biocontrol strains (1) displace the indigenous toxigenic strains, (2) persist beyond year of application; and (3) shift the indigenous A. flavus populations in a non-treated plot that is situated at a distance beyond the application site.

\section{MATERIALS AND METHODS}

\section{Fungal Strains and Inoculum Preparation}

Aspergillus flavus strains NRRL 21882, 18543 and 30797 are non-aflatoxigenic biocontrol strains that have been well characterized (Abbas et al., 2011) and sequenced (Weaver et al., 2017a,b). These strains were produced on wheat that had been moistened and autoclaved on two consecutive days, as described previously (Abbas et al., 2006). Briefly, bags containing 1,600 g of sterilized wheat were inoculated with each biocontrol strain and incubated, mixing twice per day, for 4 days before drying in a forced air drier set at $30^{\circ} \mathrm{C}$. Autoclaved, but not inoculated, wheat was also dried for use as a control treatment in field applications.

\section{Study Site}

The field experiment was conducted from 2012 through 2015 in Washington County, Mississippi (Lat: $33.43^{\circ} \mathrm{N}$; Lon: $90.94^{\circ} \mathrm{W}$ ) on a 4-ha commercial corn field in continuous corn production. The soil type was a Bosket, very fine sandy loam without irrigation. The cultural and weather conditions are described in Table 1. Field preparation included fall deep tillage and raised beds to promote drainage, followed by tillage and reshaping of the beds in the spring.

Planting involved twin rows on raised beds with $97-\mathrm{cm}$ spacing. Weed and insect pressures were closely managed to promote crop health. Plots were established by measuring $400-\mathrm{m}^{2}$ (20 rows $\times 20 \mathrm{~m}$ length) areas in April of 2012, geo-referencing the position of the plots and marking plot borders. The same plot borders were maintained throughout the 4-year study. Surface soil samples $(0-5 \mathrm{~cm}$, each a composite of five subsamples, approximately $400 \mathrm{~g}$ in total, near the center of every plot) were collected in May of each year from every plot, immediately before biocontrol applications, and once more in July, coinciding with kernel development and potential A. flavus infection. Biocontrol applications were made with hand-held fertilizer spreaders (Scotts 71,133) at a rate of $22 \mathrm{~kg} \mathrm{ha}^{-1}$, consistent with label guidelines for AflaGuard GR when corn

TABLE 1 | Growing conditions.

\begin{tabular}{|c|c|c|c|c|c|c|}
\hline \multirow[b]{2}{*}{ Year } & \multirow[b]{2}{*}{ Planting date } & \multirow[b]{2}{*}{ Hybrid planted } & \multicolumn{2}{|c|}{ Avg. Max/Min air temperature $\left({ }^{\circ} \mathrm{C}\right)^{*}$} & \multicolumn{2}{|c|}{ Precipitation $(\mathrm{mm})^{\star}$} \\
\hline & & & April-May & June-July & April-May & June-July \\
\hline 2012 & 3/20/2012 & Rev 28R10 & $28 / 16$ & $33 / 22$ & 158 & 278 \\
\hline 2013 & $4 / 10 / 2013$ & P16-15 & $24 / 13$ & $31 / 20$ & 312 & 141 \\
\hline 2014 & $3 / 21 / 2014$ & DK66-97 & $25 / 14$ & $31 / 21$ & 407 & 268 \\
\hline 2015 & $3 / 31 / 2015$ & DK62-05 & $27 / 16$ & $34 / 23$ & 317 & 136 \\
\hline Historical average & & & $26 / 14$ & $32 / 21$ & 266 & 185 \\
\hline
\end{tabular}

*Meteorological conditions recorded by Mississippi State University: http://deltaweather.extension.msstate.edu 
was at the five leaf-collar stage (V5). There were four replications of each treatment in a randomized complete block. To minimize interference between treatments, a $400-\mathrm{m}^{2}$ untreated buffer plot was included between all treatment plots. Treatments are described in Table 2, and the field layout is presented in Figure 1A. Grain was collected, by combine harvest, from the center two rows of each plot.

\section{Sample Processing and Analysis}

Soil samples were mixed with $0.1 \%$ Triton water and plated onto modified dichloran rose Bengal (MDRB) semi-selective medium to enumerate the A. flavus population (Horn and Dorner, 1998). Individual colonies were transferred to potato dextrose agar supplemented with $\beta$-cyclodextrin as a fluorescence enhancer and scored as presumptive aflatoxigenic or non-aflatoxigenic based upon production of a characteristic yellow pigment and a blue fluorescence. This methodology of Abbas et al. (2004) was used, except the scoring was done in 24-well culture dishes so that 22 isolates could be grown and scored alongside one known toxigenic strain and one known non-aflatoxigenic reference strain. This method was validated for 1,698 isolates by HPLC measurement of AF production, with the visual screen producing $89.93 \%$ accurate determination of toxigenicity.

A subsample of corn from each plot, weighing approximately $2 \mathrm{~kg}$, was ground until at least $70 \%$ of the sample passed a 20-mesh screen, as per industry protocol (Texas State Chemist). A 50-g portion was extracted with 70\% methanol for detection and quantification of $\mathrm{AF}, \mathrm{CPA}$, and fumonisin (Weaver et al., 2017a,b). An additional 20-g portion was mixed with $0.1 \%$ Triton water to isolate and characterize the A. flavus population using the same methods as the soil samples.

The colonization of the soil and grain by the non-aflatoxigenic strains was evaluated by analysis of variance (JMP 12.2, SAS Institute) at $\alpha=0.05$. For each year, the percent of non-aflatoxigenic isolates in the soil was compared between the May and July sample dates to determine the effect of biocontrol product applications in that season. No significant differences between the three biocontrol strains $(21,882,18,543$, and 30,797$)$ were detected in any year $(2012, p=0.08 ; 2013, p=0.34 ; 2014$, $p=0.34 ; 2015, p=0.75)$, so they were grouped together in the analysis. Similarly, there were no significant differences between the three biocontrol strains applied in alternating years,

TABLE 2 | Description of treatments.

\begin{tabular}{lcccc}
\hline & \multicolumn{4}{c}{ Applied inoculum } \\
\cline { 2 - 5 } Treatment & $\mathbf{2 0 1 2}$ & $\mathbf{2 0 1 3}$ & $\mathbf{2 0 1 4}$ & $\mathbf{2 0 1 5}$ \\
\hline 1. Control & Mock & Mock & Mock & Mock \\
2. Strain 21,182 & 21,182 & 21,182 & 21,182 & 21,182 \\
3. Strain 30,797 & 30,797 & 30,797 & 30,797 & 30,797 \\
4. Strain 18,543 & 18,543 & 18,543 & 18,543 & 18,543 \\
5. Alternating 21,182 & 21,182 & Mock & 21,182 & Mock \\
6. Alternating 21,182 & Mock & 21,182 & Mock & 21,182 \\
7. Alternating 30,797 & 30,797 & Mock & 30,797 & Mock \\
8. Alternating 30,797 & Mock & 30,797 & Mock & 30,797 \\
9. Alternating 18,543 & 18,543 & Mock & 18,543 & Mock \\
10. Alternating 18,543 & Mock & 18,543 & Mock & 18,543
\end{tabular}

so these treatments were also considered together. Analysis of variance and Tukey's honestly significant difference test at $\alpha=0.05$ were used to evaluate treatment effects on the A. flavus isolates that colonized grain, as measured at harvest.

\section{RESULTS AND DISCUSSION}

\section{Mycotoxin Observations}

Aflatoxin contamination is most common during periods of severe stress to the plant host, such as periods of high heat and drought, but is periodically a problem even in comparatively moist, highly productive sites (Abbas et al., 2012). This study took place during a period of historically high corn prices, leading to increased corn planting in the Mississippi Delta and supporting agronomic practices to maximize plant health. The local climatic conditions (Table 1) during our study also were favorable for corn production, as the state of Mississippi set record corn yields in 3 of the 4 years, and experienced an overall increase of $36 \%$ compared to the previous 10 -year average (NASS). These conditions were not favorable for mycotoxin contamination and we did not detect any CPA or fumonisin during this experiment. Aflatoxin was only detected in 20 of the 88 kernel samples in 2012, and only three of the 88 kernel samples exhibited AF concentrations greater than 5 ppb. In 2013, there were 13 aflatoxin-positive samples, with nine having concentrations greater than $5 \mathrm{ppb}$. Six positive samples were found in 2014, five of which were over $5 \mathrm{ppb}$. In 2015, the final year of the study, no aflatoxin was found in any of the samples. There was no significant effect of treatment on the aflatoxin concentration in the harvested corn.

\section{Colonization of Soil by A. flavus}

The percentage of aflatoxigenic isolates collected from the soil is presented in Figures 1B-F, 2. At the initiation of the experiment (Figure 1B), and before any biocontrol treatments were applied, the soil A. flavus population was determined to be about $48 \%$ (standard deviation $=15 \%)$ aflatoxigenic. Approximately 2 months after the first phase of biocontrol products were distributed in their respective plots, each treatment area had a significantly lower frequency of aflatoxigenic isolates (Figure 1C). The plots that were to receive four annual biocontrol treatments went from harboring $49 \%$ aflatoxigenic A. flavus to $11 \%$ aflatoxigenic A. flavus, and the plots to receive biennial treatments (2012 and 2014) went from $53 \%$ aflatoxigenic A. flavus to just $8 \%$. This reduction was to be expected, and is the purpose of the biocontrol application; however the co-occurrence of significant reductions in untreated plots that did not receive biocontrol treatments was not as obvious. Others have reported detection of biocontrol strains outside of the treatment area (Cotty, 1994; Bock et al., 2004). In the present study, we not only detected atoxigenic strains in untreated areas, but we observed a dramatic shift in the population in these untreated areas. Many of the "untreated" plots, scheduled to receive biocontrol treatment in subsequent years (2013 and 2015), shared a border with treated plots and may have benefited from a halo effect from an adjacent treatment. 

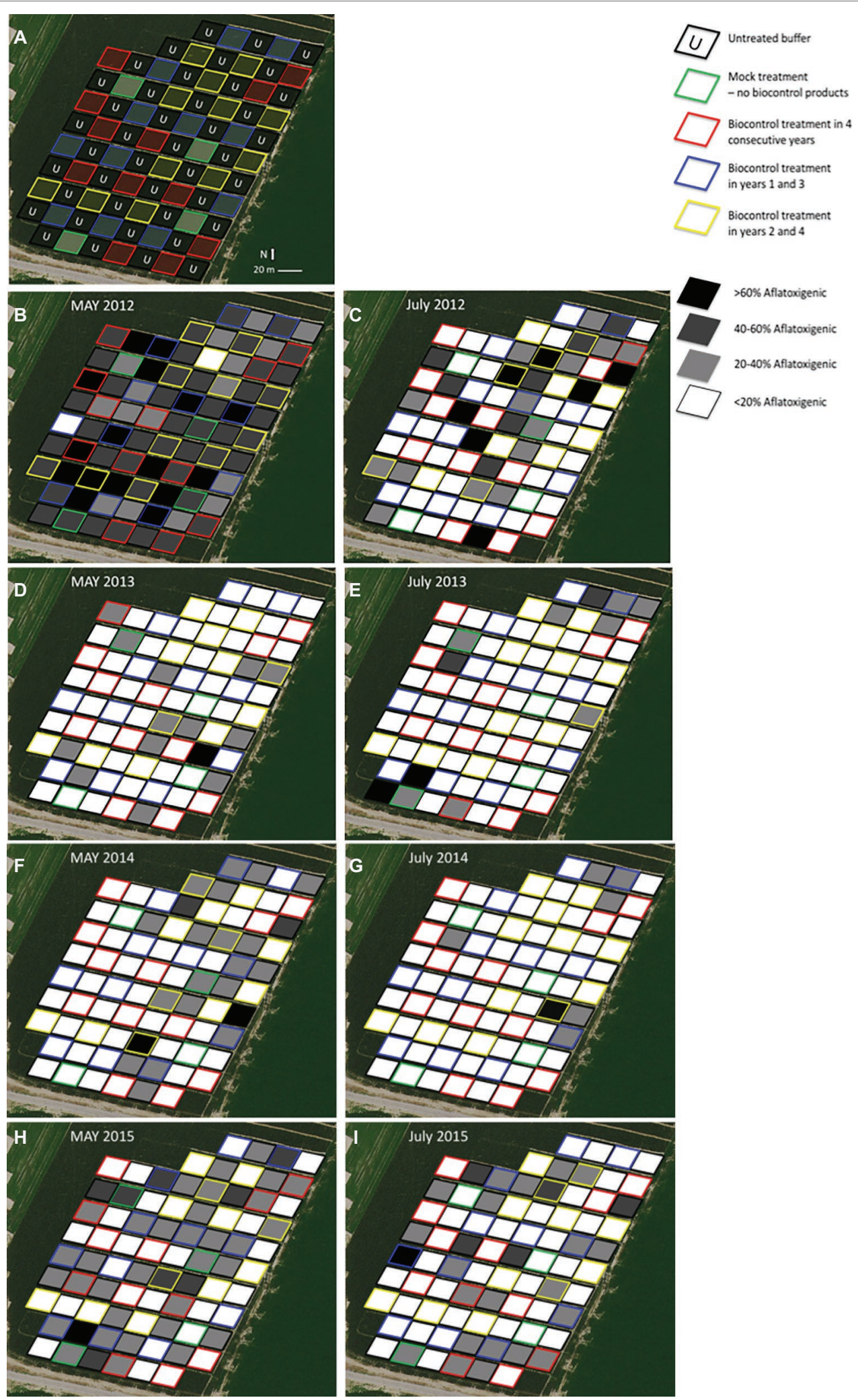

FIGURE 1 | Spatial arrangement of treatment plots within the commercial corn field and percentage of the $A$. flavus population that was aflatoxigenic within each plot at a particular time. The same plot borders were maintained throughout the 4 years of the experiment. Plot borders are color coded to indicate the treatments received and shaded to indicate the percent of $A$. flavus isolates from a plot that were aflatoxigenic. (A) clearly presents the treatments and experimental layout. (B-I) present the A. flavus population dynamics. 
The mock inoculated treatment, which only received an application of sterilized wheat, also had a highly significant aflatoxigenicity shift: between May and July, the A. flavus population in plots that received no biocontrol treatment shifted from $48 \%$ aflatoxigenic to about $30 \%$ aflatoxigenic. By the following May, less than 20\% of the strains isolated from these treatment areas were aflatoxigenic. Plots in these treatments were separated from plots with biocontrol treatments by a 20-m buffer, as indicated in Figure 1A. It is possible that the passive spread of these biocontrol strains, by normal agricultural field work, windborne spore dispersal, drift during occasional flooded conditions, or movement by insects or animals influenced the conditions of this experiment.

Plot-by-plot examination of the soil population over time reveals other patterns. In July 2012 (Figure 1C), nearly all of the plots with $>20 \%$ aflatoxigenic isolates were either untreated buffers (plots with black outlines) or plots that would not receive their first treatments until the following year (plots with yellow outlines). In 2013 and 2014 (Figures 1D-G, respectively), many of the plots with $>20 \%$ aflatoxigenic isolates were along the margins of the field; the North and South margins in 2013 and the South and East margins in 2014. If there was substantial movement of inoculum from the biocontrol treatments into nearby plots, it might be expected that plots on the edge would not shift as quickly as the central portion of the field. Results from 2015 are indicative of a shift in the population to a slightly more aflatoxigenic state, but still much less toxigenic than the initial, 2012 conditions.

The five treatments were compared for aflatoxigenicity shifts between May and July of each of the 4 years, for 20 comparisons in total (Figure 2). In the first year, as discussed above, all five treatments had a statistically significant shift toward a less toxigenic state. In the following 3 years, only five more occurrences of significant reductions in aflatoxigenic percentage were detected. Thus, of all the instances where the A. flavus population had a statistically significant shift, half of them were in the first year of treatments. The 10 occurrences of significant changes in July were also associated with comparatively highly aflatoxigenic states in May; i.e., measurable reductions in the aflatoxigenic percentage were more common when the starting population was relatively highly aflatoxigenic. Across all treatments, over the course of the entire experiment, the soil A. flavus population was $33 \%$ $($ S.E. $=5 \%)$ aflatoxigenic in May for the treatments that would have a significant reduction in the aflatoxigenic percentage by July, and just $15 \%($ S.E. $=2 \%)$ in treatments that would not have a significant reduction. A reasonable inference is that the expense of a biocontrol product application is most likely to be warranted when the initial population is highly toxigenic, and that attempting to further shift an already less toxigenic population is not as likely to be effective. It is possible that it is simply difficult to detect a meaningful shift to a lower percentage when starting from a fairly low percentage. It is also reasonable for the biocontrol products to have limited efficacy when the background population has minimal aflatoxigenicity.

\section{Colonization of Grain by A. flavus}

In 3 of the 4 years encompassed in our study, it was not possible to discern a significant effect from the biocontrol treatments on the A. flavus populations that colonized the grain (Figure 3). Only in 2014 did one of the treatments, a biennial biocontrol application, have an A. flavus population with a significantly lower percentage of aflatoxigenic isolates. This "failure" of the biocontrol, however, must be seen in context. Even if the biocontrol applications did not significantly shift the population on the grain to a less aflatoxigenic state at the time of harvest, there was still nearly zero aflatoxin observed on the corn samples. Also, in two of the years without significant effect, the background A. flavus population (the untreated or mock inoculated plots) was only $10-20 \%$ aflatoxigenic, making it impossible for us to detect any further reduction with the resolution of our analytical methods. Furthermore, this experiment did not include any of the stresses commonly associated with high aflatoxin occurrence such as extremely high temperatures, drought stress, or abundant activity by ear-feeding insects, so the full value of the biocontrol

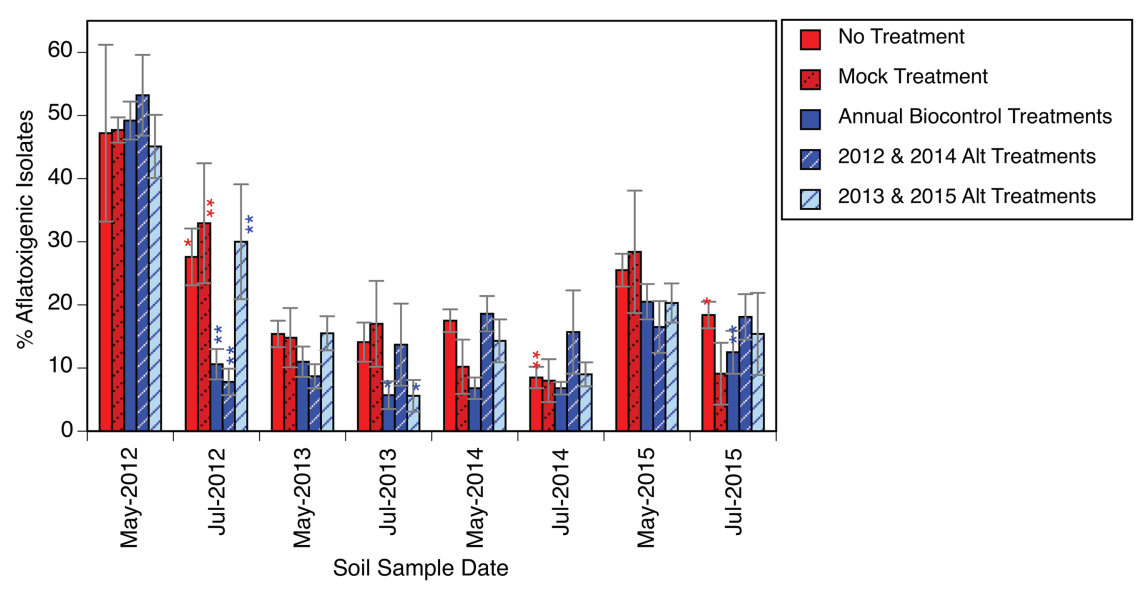

FIGURE 2 | Relative abundance of presumptive aflatoxigenic isolates in soil over time in response to biocontrol applications. Asterisk or asterisks used to indicate a significant $(\alpha=0.05)$ or highly significant $(\alpha<0.001)$ reduction, respectively in the percentage of presumptive aflatoxigenic isolates between a May and July sampling date within plots of a given treatment. Error bars indicate one standard error of the mean. 


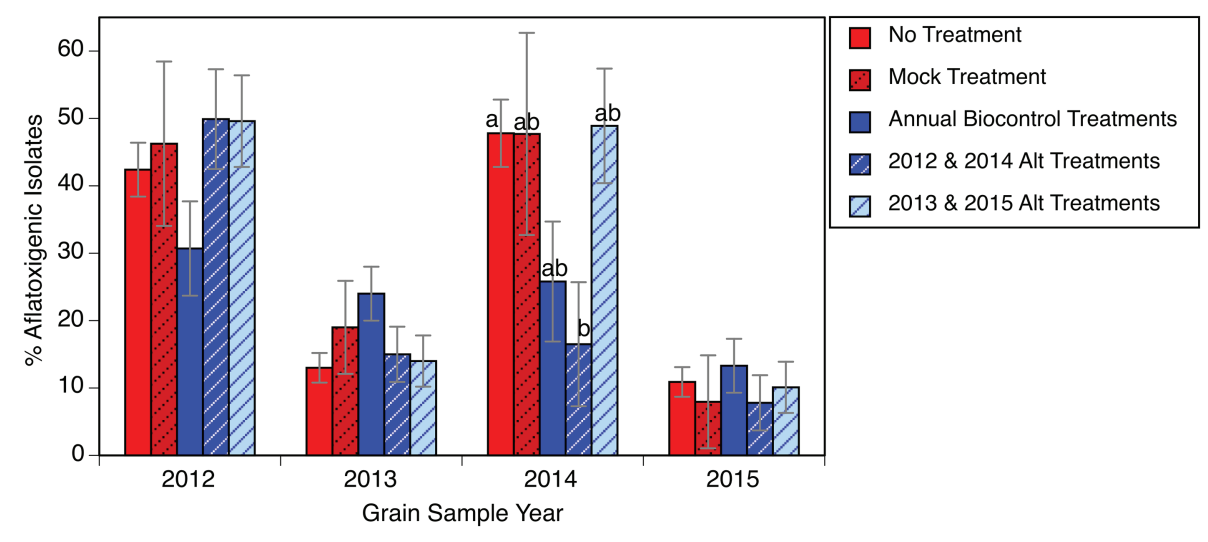

FIGURE 3 | Relative abundance of presumptive aflatoxigenic isolates in grain samples in response to biocontrol applications. Bars within the same year with different letters are significantly different by Tukey's honestly significant difference test. Error bars indicate one standard error of the mean.

applications may be somewhat masked. The reason for our observed overall increase in aflatoxigenic isolates from 2013 to 2014 is unknown and warrants further investigation. It has been suggested from vegetative compatibility group lineages that the A. flavus community has great variability from year to year, even within a given field (Bandyopadhyay et al., 2016). The frequency of aflatoxigenic isolates collected from all corn samples declined from a high of $43 \%$ (S.E. $=2.8 \%$ ) in 2012 to $11 \%$ $($ S.E. $=1.5 \%)$ in 2015 , consistent with an overall shift in the field population to a minimally aflatoxigenic level.

The experiment intended to address three hypotheses. Within the parameters of the current study, the A. flavus population in the soil shifted from 40 to $50 \%$ aflatoxigenic to consistently below $20 \%$ aflatoxigenic (hypothesis 1, Figure 2). In the final year of the experiment, there were several plots that appeared to revert from the " $<20 \%$ aflatoxigenic" to the " $20-40 \%$ aflatoxigenic" status (Figure 1). This could reflect weather conditions that year, migration of a different population, or allelic drift. Even with this change in the final year, the percentage of aflatoxigenc isolates was still less than half of the starting field average. The A. flavus population colonizing the grain from this field also declined, ending at approximately $10 \%$ aflatoxigenic (Figure 3 ). There was no evidence in the present experiment to support the expense of repeated, annual applications of biocontrol products. Similar reductions in toxigenic A. flavus were observed in plots that received biocontrol applications in alternating years (hypothesis 2). The shift to a lower frequency of aflatoxigenic isolates in untreated plots is consistent with the hypothesis that there is significant movement of inoculum (hypothesis 3). This observation could support alternative, more economical application methods.

\section{CONCLUSION}

While aflatoxin was nearly undetectable in the present experiment, it was possible to quantify the A. flavus population in soil and grain samples to monitor the decrease in the relative abundance of aflatoxigenic isolates of $A$. flavus. In the soil, significant aflatoxigenicity shifts were most common in the first year of biocontrol application. Biocontrol efficacy was positively associated with a relatively high frequency of aflatoxigenic isolates in May, immediately before application, but generally did not produce a significant effect if the background population was already $<15 \%$ aflatoxigenic. Significant population changes in untreated plots were consistent with substantial passive movement of A. flavus inoculum beyond 20 meters. After 4 years of biocontrol applications, the A. flavus population recovered from the grain was approximately $11 \%$ aflatoxigenic, regardless of the particular biocontrol treatment. Biocontrol applications are most likely to be beneficial when the initial soil population has a high percentage of aflatoxigenic isolates. Future studies should be initiated when aflatoxin levels are at greater concentrations, and also should include a single biocontrol application treatment at year one, with no other applications in subsequent years, to determine if the number of aflatoxigenic strains increases to pre-application levels.

\section{DATA AVAILABILITY}

The datasets generated for this study are available on request to the corresponding author.

\section{AUTHOR CONTRIBUTIONS}

MW planned and executed the experiment, analyzed results, and wrote the manuscript. HA participated in planning the experiment, provided technical support to sample collection, and reviewed the manuscript.

\section{ACKNOWLEDGMENTS}

The authors would like to thank Terry Johnson, Jeremy Kotowitz, Carol Morris, and Rod Patterson. Mention of trade or commercial names is solely for the purpose of providing specific information and does not imply recommendation or endorsement by the U. S. Department of Agriculture. 


\section{REFERENCES}

Abbas, H. K., Mascagni, H. J. Jr., Bruns, A., Shier, T., and Damann, K. E. (2012). Effect of planting density, irrigation regimes, and maize hybrids with varying ear size on yield, and aflatoxin and fumonisin contamination levels. Am. J. Plant Sci. 3, 1341-1354. doi: 10.4236/ajps.2012.31016

Abbas, H. K., Zablotowicz, R. M., Bruns, H. A., and Abel, C. A. (2006). Biocontrol of aflatoxin in corn by inoculation with non-aflatoxigenic Aspergillus flavus isolates. Biocontrol Sci. Tech. 16, 437-449. doi: 10.1080/09583150500532477

Abbas, H. K., Zablotowicz, R. M., Horn, B. W., Phillips, N. A., Johnson, B. J., Jin, X., et al. (2011). Comparison of major biocontrol strains of nonaflatoxigenic Aspergillus flavus for the reduction of aflatoxins and cyclopiazonic acid in maize. Food Addit. Contam. 28, 198-208. doi: 10.1080/19440049. 2010.544680

Abbas, H. K., Zablotowicz, R. M., Weaver, M. A., Horn, B. W., Xie, W., and Shier, W. T. (2004). Comparison of cultural and analytical methods for determination of aflatoxin production by Mississippi delta Aspergillus isolates. Can. J. Microbiol. 50, 193-199. doi: 10.1139/w04-006

Abbas, H. K., Zablotowicz, R. M., Weaver, M. A., Shier, W. T., Bruns, H. A., Bellaloui, N., et al. (2013). Implications of Bt traits on mycotoxin contamination in maize: overview and recent experimental results in southern United States. J. Agric. Food. Chem. 61, 11759-11770. doi: 10.1021/jf400754g

Atehnkeng, J., Ojiambo, P. S., Ikotun, T., Sikora, R. A., Cotty, P. J., and Bandyopadhyay, R. (2008). Evaluation of atoxigenic isolates of Aspergillus flavus as potential biocontrol agents for aflatoxin in maize. Food Addit. Contam., Part A 25, 1264-1271. doi: 10.1080/02652030802112635

Bandyopadhyay, R., Ortega-Beltran, A., Akande, A., Mutegi, C., Atehnkeng, J., Kaptoge, L., et al. (2016). Biological control of aflatoxins in Africa: current status and potential challenges in the face of climate change. World Mycotoxin J. 9, 771-789. doi: 10.3920/WMJ2016.2130

Bock, C. H., Mackey, B., and Cotty, P. J. (2004). Population dynamics of Aspergillus flavus in the air of an intensively cultivated region of south-West Arizona. Plant Pathol. 53, 422-433. doi: 10.1111/j.0032-0862.2004.01015.x

Bowen, K. L., Flanders, K. L., Hagan, A. K., and Ortiz, B. (2014). Insect damage, aflatoxin content, and yield of Bt corn in Alabama. J. Econ. Entomol. 107, 1818-1827. doi: 10.1603/EC13442

Brown, R. L., Cotty, P. J., and Cleveland, T. E. (1991). Reduction in aflatoxin content of maize by atoxigenic strains of Aspergillus flavus. J. Food Prot. 54, 623-626. doi: 10.4315/0362-028X-54.8.623

Bruns, H. A. (2003). Controlling aflatoxin and fumonisin in maize by crop management. J. Toxicol. Toxin Rev. 22, 153-173. doi: 10.1081/TXR-120024090

Cotty, P. J. (1994). Influence of field application of an atoxigenic strain of Aspergillus flavus on the populations of A. flavus infecting cotton bolls and on the aflatoxin content of cottonseed. Phytopathology 84, 1270-1277. doi: 10.1094/Phyto-84-1270

Diener, U. L., Cole, R. J., Sanders, T. H., Payne, G. A., Lee, L. S., and Klich, M. A. (1987). Epidemiology of aflatoxin formation by Aspergillus flavus. Annu. Rev. Phytopathol. 25, 249-270. doi: 10.1146/annurev.py.25.090187.001341

Dorner, J. W. (2010). Efficacy of a biopesticide for control of aflatoxins in corn. J. Food Prot. 73, 495-499. doi: 10.4315/0362-028X-73.3.495

Dowd, P. F. (2001). Biotic and abiotic factors limiting efficacy of Bt corn in indirectly reducing mycotoxin levels in commercial fields. J. Econ. Entomol. 94, 1067-1074. doi: 10.1603/0022-0493-94.5.1067
Horn, B. W., and Dorner, J. W. (1998). Soil populations of Aspergillus species from section Flavi along a transect through peanut-growing regions of the United States. Mycologia 90, 767-776.

IARC (1993). "Some naturally occurring substances: food items and constituents, heterocyclic aromatic amines and mycotoxins" in International agency for research on cancer: Monographs on the evaluation of carcinogenic risks to humans, vol. 56 (Lyon, France: IARC Press), 1-599. Available from: http:// monographs.iarc.fr/ENG/Monographs/vol56/index.php

Jones, R. K., Duncan, H. E., and Hamilton, P. B. (1981). Planting date, harvest date, and irrigation effects on infection and aflatoxin production by Aspergillus flavus in field corn. Phytopathology 71, 810-816. doi: 10.1094/ Phyto-71-810

Mauro, A., Garcia-Cela, E., Pietri, A., Cotty, P. J., and Battilani, P. (2018). Biological control products for aflatoxin prevention in Italy: commercial field evaluation of atoxigenic Aspergillus flavus active ingredients. Toxins 10:30. doi: 10.3390/toxins10010030

Mitchell, N. J., Bowers, E., Hurburgh, C., and Wu, F. (2016). Potential economic losses to the US corn industry from aflatoxin contamination. Food Addit. Contam. Part A 33, 540-550. doi: 10.1080/19440049.2016.1138545

Munkvold, G. P., Hellmich, R. L., and Rice, L. G. (1999). Comparison of fumonisin concentrations in kernels of transgenic $\mathrm{Bt}$ maize hybrids and nontransgenic hybrids. Plant Dis. 83, 130-138. doi: 10.1094/PDIS.1999.83.2.130

Payne, G. (1992). Aflatoxin in maize. Crit. Rev. Plant Sci. 10, 423-440. doi: 10.1080/07352689209382320

Weaver, M. A., Abbas, H. K., Brewer, M. J., Pruter, L. S., and Little, N. S. (2017a). Integration of biological control and transgenic insect protection for mitigation of mycotoxins in corn. Crop Prot. 98, 108-115. doi: 10.1016/J. CROPRO.2017.03.020

Weaver, M. A., Abbas, H. K., Falconer, L. L., Allen, T. W., Pringle, H. C., and Sciumbato, G. L. (2015). Biological control of aflatoxin is effective and economical in Mississippi field trials. Crop Prot. 69, 52-55. doi: 10.1016/j. cropro.2014.12.009

Weaver, M. A., Scheffler, B. E., Duke, M., Ballard, L., Abbas, H. K., and Grodowitz, M. J. (2017b). Genome sequences of three strains of Aspergillus flavus for the biological control of aflatoxin. Genome Announc. 5, e01204-e01217. doi: 10.1128/genomeA.01204-17

Wiatrak, P. J., Wright, D. L., Marois, J. J., and Wilson, D. (2005). Influence of planting date on aflatoxin accumulation in $\mathrm{Bt}$, non-Bt, and tropical non-Bt hybrids. Agron. J. 97, 440-445. doi: 10.2134/agronj2005.0440

Wild, C. P., Miller, J. D., and Groopman, J. D. (2015). Mycotoxin control in low- and middle-income countries, vol. 9. Lyon, France: International Agency for Research on Cancer: Working Group.

Conflict of Interest Statement: The authors declare that the research was conducted in the absence of any commercial or financial relationships that could be construed as a potential conflict of interest.

Copyright (C) 2019 Weaver and Abbas. This is an open-access article distributed under the terms of the Creative Commons Attribution License (CC BY). The use, distribution or reproduction in other forums is permitted, provided the original author(s) and the copyright owner(s) are credited and that the original publication in this journal is cited, in accordance with accepted academic practice. No use, distribution or reproduction is permitted which does not comply with these terms. 